\title{
Computer-Based Cognitive Stimulation Intervention
}

National Cancer Institute

\section{Source}

National Cancer Institute. Computer-Based Cognitive Stimulation Intervention. NCI

Thesaurus. Code C147860.

Computer-based programs designed to improve thinking, concentration, memory, and communication. 